\title{
A case of poorly differentiated adenocarcinoma with lymphoid stroma originated in the ascending colon diagnosed as lymphoepithelioma-like carcinoma
}

\author{
Kengo Kai ${ }^{1,3} \cdot$ Hideki Hidaka $^{1} \cdot$ Takeshi Nakamura $^{1} \cdot$ Yuji Ueda $^{1} \cdot$ Kosuke Marutsuka $^{2} \cdot$ Takuto Ikeda $^{3} \cdot$ \\ Atsushi Nanashima ${ }^{3}$
}

Received: 20 August 2019 / Accepted: 4 December 2019 / Published online: 16 December 2019

(c) The Author(s) 2019

\begin{abstract}
An 86-year-old woman's stool sample was positive for blood. Computed tomography (CT) showed wall thickening of the ascending colon at the hepatic flexure. Colonoscopy showed near-complete obturation by colon cancer. Since she was asymptomatic, elective surgery was planned. Laparoscopic right hemicolectomy was performed. Histopathological examination showed poorly differentiated carcinoma cells proliferating in a solid pattern with marked lymphocyte infiltration. The diagnosis was lymphoepithelioma-like carcinoma (LELC) associated with Epstein-Barr virus (EBV) infection; however, EBV-encoded small RNA-in situ hybridization was negative. Microsatellite instability was not assessed. The postoperative course was uneventful and she was discharged on the 15th postoperative day. She remains recurrence-free at 2 years after surgery. Past reports note that colorectal carcinomas with dense lymphoid stroma may be related to LELC or medullary carcinoma (MC). Gastrointestinal LELC is rare, with some reports on LELC of the esophagus and stomach. Reports on LELC of the large intestine are very rare. MC of the large intestine is relatively new concept, firstly described in the WHO Classification of Tumours of the Digestive System 3rd Edition in 2000. We herein present a case of lymphoepithelioma-like carcinoma of the ascending colon and relevant case reports about LELC and MC of the large intestine.
\end{abstract}

Keywords Carcinoma with lymphoid stroma $\cdot$ Ascending colon cancer $\cdot$ Lymphoepithelioma-like carcinoma $\cdot$ Medullary carcinoma $\cdot$ EBER-ISH (EBV-encoded small RNA-in situ hybridization)

\section{Introduction}

Poorly differentiated adenocarcinoma associated with dense lymphoid stroma may be associated with two subsets: lymphoepithelioma-like carcinoma (LELC), which is associated with Epstein Barr virus (EBV) infection; and medullary carcinoma (MC), which is associated with high

Kengo Kai

kengo_kai@med.miyazaki-u.ac.jp

1 Department of Surgery, Miyazaki Prefectural Miyazaki Hospital, 5-30 Kitatakamatsu, Miyazaki City, Miyazaki 8808510, Japan

2 Department of Pathology, Miyazaki Prefectural Miyazaki Hospital, 5-30 Kitatakamatsu, Miyazaki City, Miyazaki 8808510, Japan

3 Department of Surgery, Faculty of Medicine, University of Miyazaki, 5200 Kihara, Kiyotake, Miyazaki City, Miyazaki 8891692, Japan microsatellite instability (MSI-H) [1]. LELC occurring in the gastrointestinal tract is rare, with some reports describing the involvement of the esophagus and stomach. Reports on colon involvement are very scarce. MC of the large intestine was first described in the World Health Organization Classification of Tumours of the digestive system 3rd Edition in 2000 [2]. Reported cases are uncommon, similarly to LELC of the large intestine. Both types are associated with a good prognosis in comparison to poorly differentiated colorectal carcinoma. We herein report a case of poorly differentiated adenocarcinoma with lymphoid stroma of the ascending colon that was diagnosed as LELC based on the similarity to LELC involving other organs, such as stomach or lung. We also review past case reports on LELC and MC of the large intestine. 


\section{Case report}

The patient was an 86-year-old woman who was initially managed by a general practitioner. She underwent colonoscopy after blood was detected in a stool sample. Colonoscopy revealed circumferential cancer in the ascending colon. The colonic lumen was almost obturated and the scope could not pass through the tumor to the oral side (Fig. 1). The patient was transferred to our hospital for surgical treatment. She did not have a family history and past history of malignant disease. The patient was not in good health and had been diagnosed with dementia and disuse syndrome after lumber compression fracture. An abdominal examination revealed a palpable mass without tenderness. With the exception of anemia (hemoglobin, $11.7 \mathrm{~g} / \mathrm{dL}$ [normal range: $13.2-17.2 \mathrm{~g} / \mathrm{dL}$ ]), the patient's laboratory findings ware unremarkable, including tumor marker levels such as carcinoembryonic antigen (CEA), carbohydrate antigen 19-9 (CA19-9). Enhanced computed tomography (CT) revealed wall thickening with the enhancement of the ascending colon at the hepatic flexure without the findings suggesting lymph node metastasis, distant metastasis or intestinal obstruction (Fig. 2).

These findings led to a diagnosis of advanced ascending colon cancer of T3N0M0 cStage IIA, according to the International Union Against Cancer TNM Classification(UICC), 8th edition. Since she had no symptoms, elective surgery was planned for 12 days after admission. Laparoscopic right hemicolectomy and D3 lymph node dissection were performed. Ileocolonic

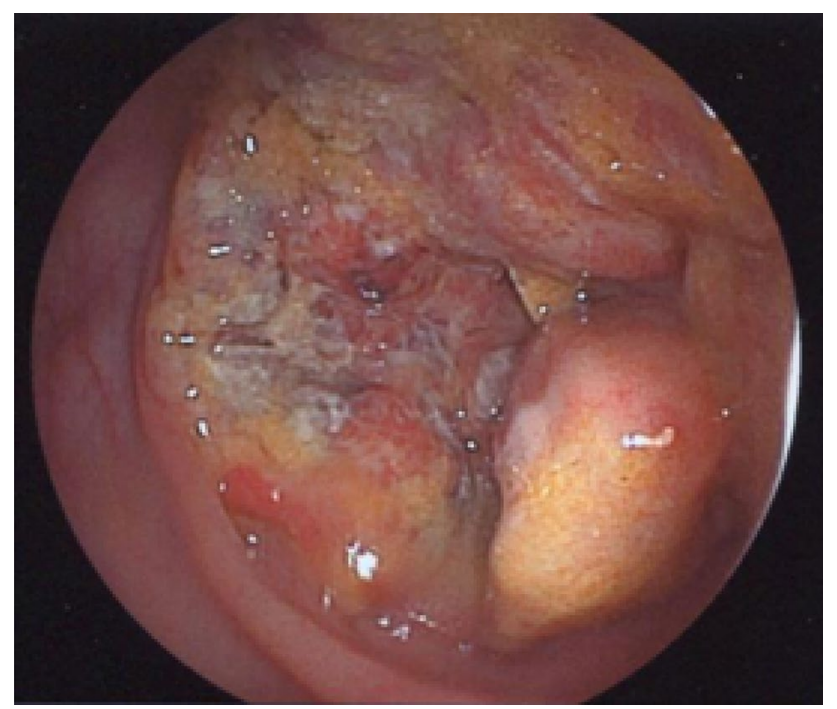

Fig. 1 Colonoscopy: circumferential cancer located in the ascending colon almost obturated the colonic lumen and the scope could not pass through the tumor to the oral side

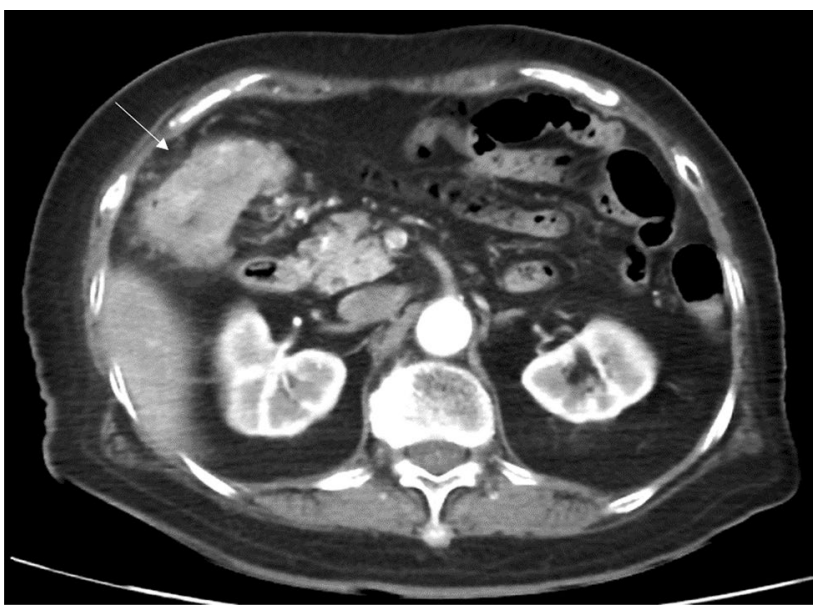

Fig. 2 Enhanced computed tomography: wall thickening with enhancement at the hepatic flexure (arrow) without the findings suggesting lymph node metastasis, distant metastasis and intestinal bowel obstruction

anastomosis was reconstructed with functional end-to-end anastomosis technique.

The resected specimen revealed a nodule-aggregated lesion of $75 \times 63 \mathrm{~mm}$ in size (Fig. 3). Hematoxylin and eosin staining showed that tumor demonstrated nodular growth, occupying the entire wall in low power view (Fig. 4a) and that the undifferentiated tumor cells with large pleomorphic nuclei and some small nucleoli proliferate diffusely without cohesiveness, accompanied by diffuse lymphoplasmacytic infiltration in the tumor in high power view (Fig. 4b). Immunostaining revealed to be predominantly composed of T-lymphocytes (Fig. 5b). Thus, it was believed to be a primary colorectal LELC with dense lymphoid stroma. The tumor exhibited no lymphatic invasion and was positive for por1, pSS, int, INFc, ly2, v1, PM0, DM0, RM0. An immunohistological assessment of the tumor cells using EBVencoded small RNA- in situ hybridization was negative for EBV (Fig. 5c).

The patient's postoperative course was uneventful and she was discharged on the 15 th postoperative day. No evidence of cancer recurrence has been seen during 2 years of followup without adjuvant chemotherapy after the surgery.

\section{Discussion}

We described a case of poorly differentiated adenocarcinoma with lymphoid stroma originated in the ascending colon that was diagnosed as LELC. The term "lymphoepithelioma" was coined in 1921 by Regaud and Reverchon [3] and Schminke [4] to describe a tumor occurring in the nasopharynx, related to EBV infection. This tumor is characteristically composed of cells with large vesicular nuclei, 
Table 1 Reported cases of lymphoepithelioma-like carcinoma of the large intestine including our case (10 cases)

\begin{tabular}{lllllllllllll}
\hline Case no & Author & Year & Age & Sex & Site & $\begin{array}{l}\text { Tumor } \\
\text { size }(\mathrm{mm})\end{array}$ & T & N & M & MSI assesment & EBER-ISH in tumor cells \\
\hline 1 & Vilor [12] & 1995 & 77 & F & T & 120 & 3 & 1 & 0 & Not described & Negative \\
2 & Palazzo [13] & 1995 & 29 & M & R & 35 & 3 & - & - & Not described & Negative \\
3 & Samaha [14] & 1998 & 62 & M & C & 28 & 3 & 1 & - & Not described & Not described \\
4 & De Petris [15] & 1999 & 44 & M & A & 9 & 1 & - & - & Not performed (HNPCC) & Negative \\
5 & Kon [16] & 2001 & 72 & M & R & 25 & 2 & 0 & - & Not described & Positive \\
6 & Kojima [17] & 2006 & 25 & M & R & 7 & 1 & 0 & 0 & Not described & Weak positive \\
7 & Taniguchi [18] & 2011 & 88 & F & T & 40 & 3 & 0 & - & Not described & Negative \\
8 & Delaney [19] & 2012 & 85 & F & S & 42 & 2 & 0 & - & Loss of MLH-1 and PMS-2 & Negative \\
9 & Mori [20] & 2013 & 70 & F & A & 70 & 1 & 0 & - & Not described & Negative \\
10 & Our case & 2019 & 86 & F & T & 75 & 3 & 0 & 0 & Not performed & Negative \\
\hline
\end{tabular}

MSI microsatellite instability, EBER-ISH EBV-encoded small RNA-in situ hybridization, HNPCC hereditary non-polyposis colorectal cancer

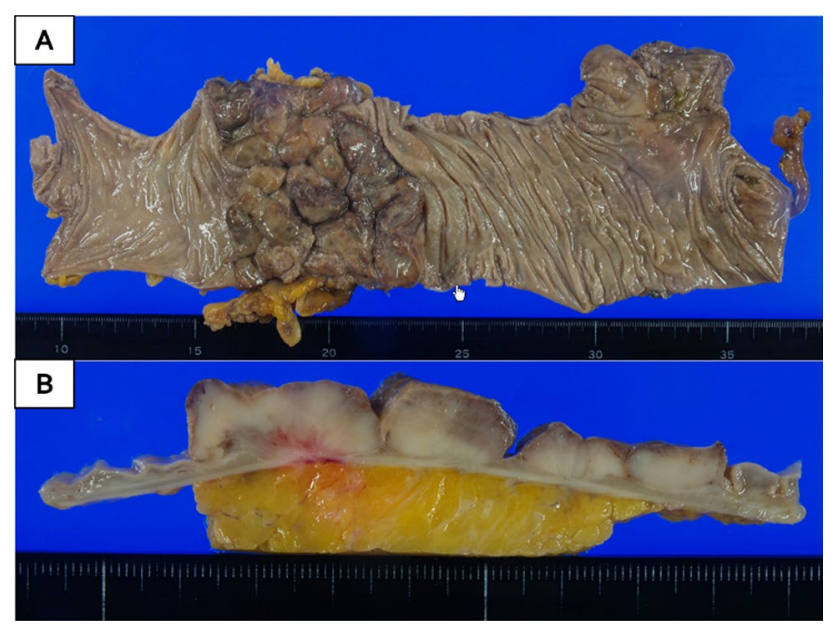

Fig. 3 Macroscopic examination: The resected specimen showed type 1 cancer resembling like a nodule-aggregated lesion, which measured $75 \times 63 \mathrm{~mm}$ in size $(\mathbf{a}, \mathbf{b})$

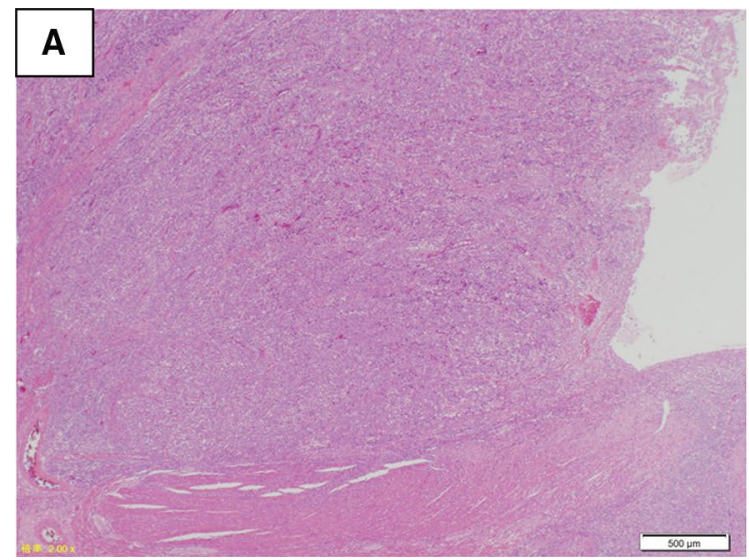

Fig. 4 Microscopic findings (Hematoxylin and eosin stain): In low power view, tumor shows nodular growth, occupying the entire wall (a). In the high power view, the undifferentiated tumor cells with single prominent nucleoli, and indistinct cell borders, which impart a syncytial appearance to the tumor, and has an attendant lymphoplasmacytic inflammatory component [5]. With this hallmark, LELC has been described at various locations outside the nasopharynx, including the thymus, salivary gland, lung, vagina, skin, urinary bladder and mammary gland; however, the majority of these cases were not associated with EBV. LELC occurring in the gastrointestinal tract is uncommon, and the best recognized example of LELC in the gastrointestinal tract occurs in the stomach. LELC of the stomach was first described by Watanabe et al. in 1976 as "gastric carcinoma with a lymphoid stroma" [6]. EBV is associated with 5-20\% of gastric carcinomas worldwide [7-9]; however, more than $80 \%$ of LELCs of the stomach have been found to be related to EBV infection [10] in comparison to only approximately $6-7 \%$ of non-LELC [11].

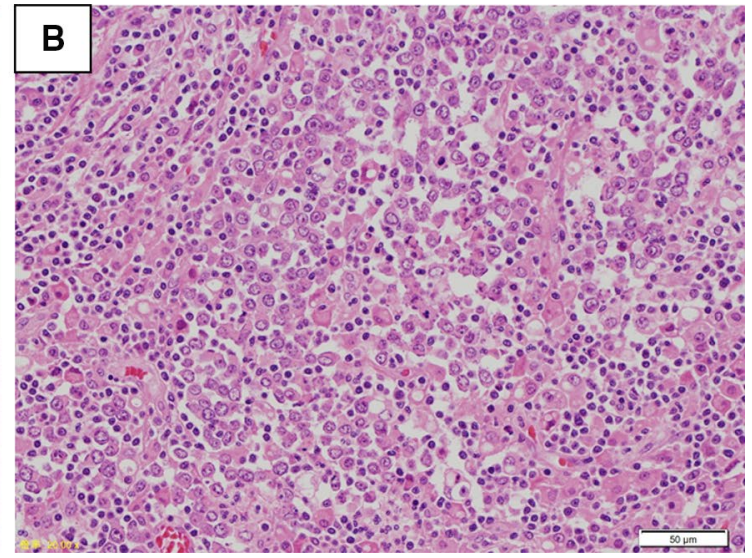

large pleomorphic nuclei and some small nucleoli proliferate diffusely without cohesiveness, accompanied by diffuse lymphoplasmacytic infiltration in the tumor (b) 

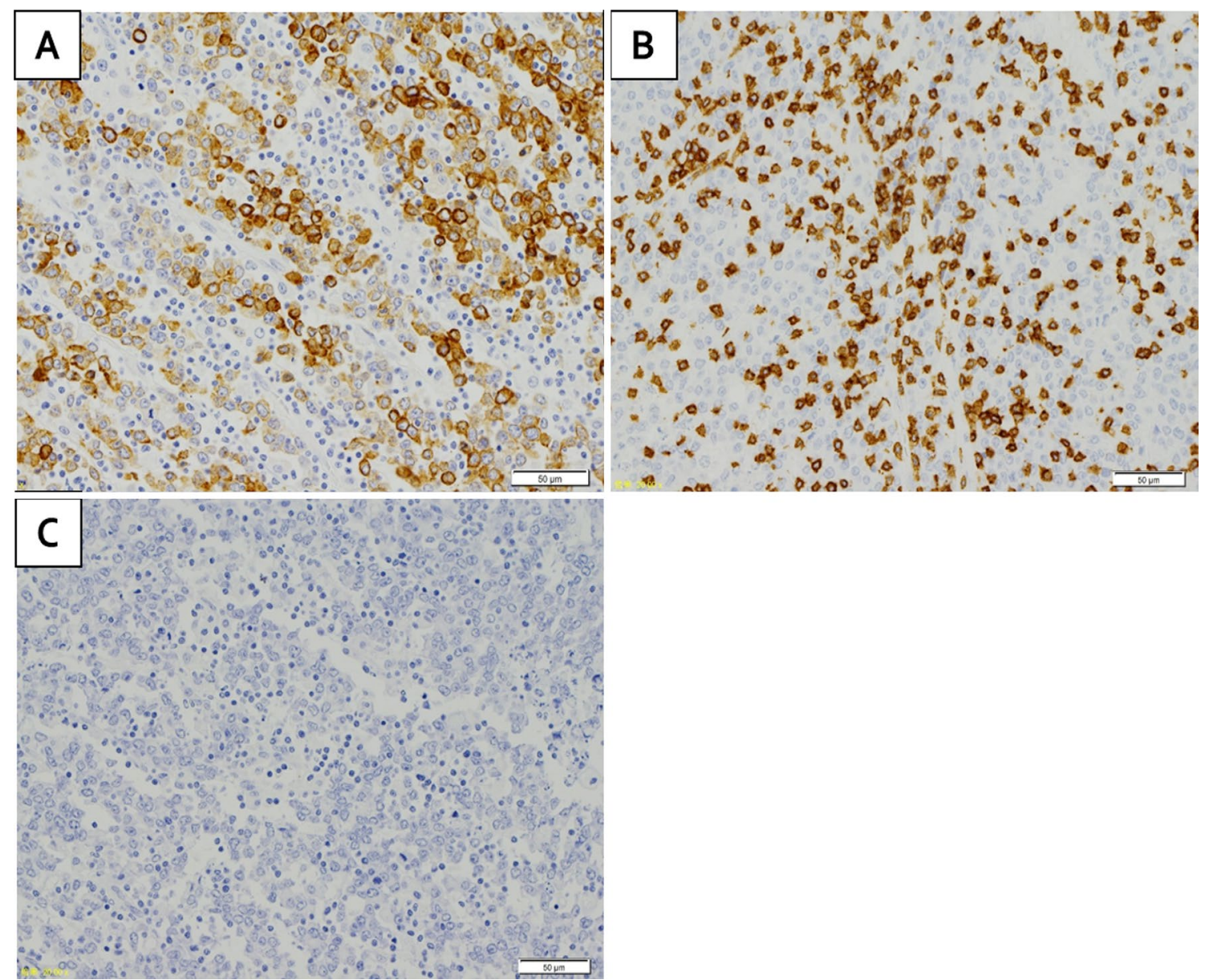

Fig. 5 Pathologic findings (Immunohistochemical staining): a positive staining for AE1/AE3 in LELC. b Positive staining for CD3 in the lymphocytes. EBV-encoded small RNA-in situ hybridization (EBER-ISH) revealed that tumor cells and lymphocytes were negative for EBV

Table 2 Reported cases of medullary carcinoma of the large intestine (10 cases)

\begin{tabular}{lllllllllllll}
\hline Case no & Author & Year & Age & Sex & Site & $\begin{array}{l}\text { Tumor } \\
\text { size }(\mathrm{mm})\end{array}$ & T & N & M & MSI-H assess ment & EBER-ISH in tumor cell \\
\hline 1 & Wang [25] & 2005 & 79 & F & A & 90 & - & 1 & 0 & Not described & Not described \\
2 & Sakurai [26] & 2012 & 78 & F & T & 20 & 1 & 0 & 0 & Loss of MLH-1 and PMS-2 & Not described \\
3 & Cunningham [27] & 2014 & 79 & F & A & 60 & 3 & 1 & 1 & Loss of MLH-1 and PMS-2 & Not described \\
4 & Cunningham [27] & 2014 & 81 & F & T & - & 4 & 0 & 0 & Loss of MLH-1 and PMS-2 & Not described \\
5 & Mitchell [28] & 2014 & 75 & F & C & 40 & 2 & 2 & 0 & Loss of MLH-1 and PMS-2 & Negative \\
6 & Jain [29] & 2014 & 72 & F & D & 80 & 2 & 0 & 0 & Loss of MLH-1 and PMS-2 & Not described \\
7 & Kasapidis [30] & 2015 & 58 & M & A & - & 3 & 1 & 0 & Not described & Not described \\
8 & Wakasugi [31] & 2017 & 72 & F & T & 60 & 3 & 0 & 0 & Loss of MLH-1 and CDX-2 & Not described \\
9 & Martinotti [32] & 2017 & 44 & F & A & 60 & 3 & 0 & 0 & Loss of MLH-1 and CDX-2 & Negative \\
10 & Yago [33] & 2018 & 63 & M & A & 75 & 2 & 0 & 0 & Loss of MLH-1 & Not described
\end{tabular}

MSI microsatellite instability, EBER-ISH EBV-encoded small RNA-in situ hybridization

With regards to colorectal LELC, thus far only 10 cases have been reported, including our case [12-20] (Table 1). According to previous case reports, LELC of the large intestine is diagnosed based on the pathological similarity to LELC of other organ. The tumor cells are large, oval or polygonal in shape, contain vesicular to clear nuclei, 
prominent nucleoli, and abundant eosinophilic cytoplasm with poorly defined cell borders [21, 22]. The small lymphocytes can infiltrate cancer cell nests and epithelioid granulomas are sometimes observed within the lymphoid stroma. The lymphoid reaction is composed predominantly of T-cells, including cytotoxic lymphocytes and/or natural killer cells in close contact with tumor cells [23]. The mechanism by which EBV contributes to the lymphocytic reaction for LELC is still unknown. Poorly differentiated adenocarcinoma is the most frequent type of LELC. However, LELC is known to be relatively less aggressive (clinically), despite its poorly differentiated features [24].

The other type of the colorectal cancer with dense lymphoid stroma is medullary carcinoma (MC). Colorectal MC is a rare disease, only 10 cases reported in the literature to date; colorectal MC is almost always associated with a microsatellites instability-high (MSI-H) status [25-33] (Table 2). Thirunavukarasu et al. [34] reported that MC was a rare tumor, accounting for approximately 5-8 of every 10,000 colon cancers diagnosed. Similarly to LELC, MC should be distinguished from other more aggressive colorectal cancers because MC appears to be associated with better survival outcomes than undifferentiated colon adenocarcinoma. Patients with MC commonly present with Stage II disease, with $10 \%$ presenting metastasis. An analysis of the early outcomes in patients with MC revealed that 1- and 2- year relative survival rates of $92.7 \%$ and $73.8 \%$, respectively [34]. MC is morphologically characterized by sheets of malignant cells with vesicular nuclei, prominent nucleoli and abundant eosinophilic cytoplasm, as well as the prominent infiltration of tumoral lymphocytes. MC is known to be associated with MSI-H, and some studies examining the immunohistochemical characteristics of this tumor have shown that it can be differentiated from poorly differentiated colorectal adenocarcinoma by a loss of MLH-1 staining and the intestinal transcription factor CDX2.

Colorectal cancer with MSI-H is classified into two subtype; hereditary non-polyposis colorectal cancer (HNPCC) typically occurs in the youth, while sporadic colorectal cancer with MSI-I typically occurs in the elderly [35]. The mean age at the diagnosis of MC of the large intestine is reported to be 69 years, with the incidence increasing with age [34]. HNPCC is characterized by an autosomal dominant inherited pattern of susceptibility to colorectal cancer at an early age, the multiplicity of colorectal cancer, proximal tumor location and potential carcinogenesis of the endometrium and other organs [36]. HNPCC is diagnosed based on stringent clinical criteria (the Amsterdam criteria) and germline mutations in mismatch repair genes associated with MSI-H, such as MLH1, MSH2, PMS1 and PMS2 [37]. Interestingly, De Petris et al. [15] reported a case of LELC of the colon in a patient with HNPCC. From the description of the tumor, it would appear to conform to the morphological criteria for an LELC rather than an MC. Furthermore, the patient was negative for EBV infection. Because of the family history and the pathologic feature, the patient was believed to be affected by HNPCC. The diagnosis of HNPCC was confirmed in a center for hereditary gastrointestinal cancer; however, the patient refused to undergo genetic testing.

In a review article, Chetty et al. [1] noted that MC demonstrates a syncytial growth pattern, bears robust peritumoral inflammation, and has a well-defined border, while LELC is formed by small clusters of cells that "do not correspond to the syncytial growth patterns seen in MC", has more prominent intratumoral than peritumoral inflammation, and has an infiltrative border. On the other hand, Gonzalez et al. [38] reported that these tumors are often classified together, thereby implying that they are the same entity. They do not recommend relying on histologic criteria alone to distinguish between LELC and MC, because of the overlap in syncytial and clustered architecture and the fact that intratumoral and peritumoral inflammation are not mutually exclusive. Thus, they report that the term, "carcinoma with lymphoid stroma", should best serve as a categorizing diagnosis after the initial histological review. Determining the EBV and MSI status via ancillary testing currently appears to be the most reliable way to arrive at a final diagnosis.

In this case, the pathologist in our institution diagnosed poorly differentiated carcinoma with lymphoid stroma with LELC based on morphologic features, which were similar to LELC of other organs, especially in terms of intratumoral-dominant inflammation and an undefined border. While we considered performing an additional assessment to determine the MSI status, we did not perform the assessment because the possibility of HNPCC was considered to be very low based on the patient's age and family history. Of course, it is interesting to concern the pathogenesis of the carcinoma with molecular status, but it seems that it be more important to keep in mind the possibility of HNPCC in colon carcinoma with lymphoid stroma and to interview the patient based on Amsterdam criteria according to the age. The past cases suggest that when a physician encounters colon carcinoma with a dense lymphoid stroma, it is clinically important to perform an assessment for HNPCC, regardless of whether LELC or $\mathrm{MC}$ is diagnosed based on the histological findings.

Funding None of the authors have anything to disclose.

\section{Compliance with ethical standards}

Conflict of interest The authors, Kengo kai, Hideki Hidaka, Takeshi Nakamura, Yuji Ueda, Kosuke Marutsuka, Takuto Ikeda and Atsushi Nanashima, declare that they have no conflict of interests. 
Ethical approval All procedures used in this research have been performed in accordance with the ethical standard laid down in the 1964 Declaration of Helsinki and its later amendments.

Informed consent Informed consent was obtained for using the clinical information and accompanying image as research activities.

Open Access This article is licensed under a Creative Commons Attribution 4.0 International License, which permits use, sharing, adaptation, distribution and reproduction in any medium or format, as long as you give appropriate credit to the original author(s) and the source, provide a link to the Creative Commons licence, and indicate if changes were made. The images or other third party material in this article are included in the article's Creative Commons licence, unless indicated otherwise in a credit line to the material. If material is not included in the article's Creative Commons licence and your intended use is not permitted by statutory regulation or exceeds the permitted use, you will need to obtain permission directly from the copyright holder. To view a copy of this licence, visit http://creativecommons.org/licenses/by/4.0/.

\section{References}

1. Chetty R. Gastrointestinal cancers accompanied by dense lymphoid component: an overview with special reference to gastric and colonic medullary and lymphoepithelioma-like carcinomas. J Clin Pathol. 2012;65:1062-5.

2. Hamilton SR, Vogelstein B, Hudos S, et al. Tumours of the colon and rectum -Carcinoma. In Hamilton SR, Aaltonen LA, editors. Pathology and Genetics of Tumours of the Digestive System. World Health Organization Classification of Tumours. Lyon: IARC Press; 2000. pp 105-119.

3. Regaud C, Reverchon L. Sur un cas d'epithliome epidermoide developpe dams les massif maxillaire superieur. Rev Laryngol Otol Rhinol. 1921;42:369-78 (in French).

4. Schminke A. Uber lympho-epitheliale Geschwulste. Beitr Pathol Anat Allg Pathol. 1921;68:161-70 (in German).

5. Burke AP, Yen TSB, Shekitka KM, et al. Lymphoepithelioma-like carcinoma of the stomach with Epstein-Barr virus demonstrated by polymerase chain reaction. Mod Pathol. 1990;3:377-80.

6. Watanabe H, Enjoji M, Imai T. Gastric carcinoma with lymphoid stroma Its morphologic characteristic and prognostic correlations. Cancer. 1976;38:232-43.

7. Fukayama M, Hayashi Y, Iwasaki Y, et al. Epstein-Barr virus associated gastric carcinoma and Epstein-Barr virus infection of the stomach. Lab Invest. 1994;71:73-81.

8. Rowlands DC, Ito M, Mangham DC, et al. Epstein-Barr virus and carcinoma: rare association of the virus with gastric adenocarcinoma. Br J Cancer. 1993;68:1014-9.

9. Shibata D, Weiss LM. Epstein-Barr virus associated gastric adenocarcinoma. Am J Pathol. 1992;140:769-74.

10. Matsunou H, Konishi F, Hori H, et al. Characteristics of EpsteinBarr virus associated gastric carcinoma with lymphoid stroma in Japan. Cancer. 1996;77:1998-2004.

11. Torlakovic G, Snover DC, Torlakovic E. Simultaneous EBV-positive lymphoepithelioma-like carcinoma and EBV-negative intestinal type adenocarcinoma in a patient with Helicobacter pyloriassociated chronic gastritis. Am J Clin Pathol. 2004;121:237-43.

12. Vilor M, Tsutsumi Y. Localization of Epstein-Barr genome in lymphoid cells in poorly differentiated adenocarcinoma with lymphoid stroma of the colon. Pathol Int. 1995;45:695-7.

13. Palazzo JP, Mittal KR. Lymphoepithelioma-like carcinoma of the rectum in a patient with ulcerative colitis. Am J Gastroenterol. 1996;91:398-9.
14. Samaha S, Tawfik O, Horvat R, et al. Lymphoepithelioma-like carcinoma of the colon: report of a case with histologic, immunohistochemical, and molecular studies for Epstein-Barr virus. Dis colon Rectum. 1998;41:925-8.

15. De Petris G, Lev R, Quirk DM, et al. Lymphoepithelioma-like carcinoma of the colon in a patient with hereditary nonpolyposis colorectal cancer. Arch Pathol Lab Med. 1999;123:720-4.

16. Kon S, Kasai K, Tsuzuki N, et al. Lymphoepithelioma-like carcinoma of rectum: possible relation with EBV. Pathol Res Pract. 2001;197:577-82.

17. Kojima Y, Mogaki M, Takagawa R, et al. A case of lymphoepithelioma-like carcinoma of the colon with ulcerative colitis. J Gastroenterol. 2007;42:181-5.

18. Taniguchi R, Nagata Y, Niino D, et al. Lymphoepithelial-like carcinoma of the colon. Jpn J Gastroenterol Surg. 2011;44:591-5.

19. Delaney D, Chetty R. Case report: Lymphoepithelioma-like carcinoma of the colon. Int J Clin Exp Pathol. 2012;5:105-9.

20. Mori Y, Akagi K, Yano H, et al. Lymphoepithelioma-like carcinoma of the colon. Case Rep Gastroenterol. 2013;7:127-33.

21. Min K, Holmquist S, Peiper SC, et al. Poorly differentiated adenocarcinoma with lymphoid stroma (lymphoepithelioma-like carcinoma) of the stomach. Am J Clin Pathol. 1991;96:2219-27.

22. Minamoto T, Mai M, Watanabe K, et al. Medullary carcinoma with lymphocytic infiltration of the stomach, clinicopathological study of 27 cases and immunohistochemical analysis of the subpopulations of infiltrating lymphocytes in the tumour. Cancer. 1990;66:945-52.

23. Chapel F, Fabiani B, Davi F, et al. Epstein-Barr virus and gastric carcinoma in Western patients: comparison of pathological parameters and p53 expression in EBV-positive and negative tumours. Histopathology. 2000;36:252-61.

24. Nacopoulou L, Azaris P, Papacharalampous N, et al. Prognostic significance of histologic host response in cancer of the large bowel. Cancer. 1981;47:930-6.

25. Wang L, Warner NE, Sherrod AE. Pathological quiz case: a 79-year-old woman with a black, ulcerated cecal tumor and 3 negative guaiac test results. Arch Pathol Lab Med. 2005;129:113-4.

26. Sakurai U, Saito M, Mitsui H, et al. Early-stage medullary type poorly differentiated adenocarcinoma of the transverse colon. Stomach Intestine. 2012;47:1865-71.

27. Cunningham J, Kantekure K, Saif MW. Medullary carcinoma of the colon: a case series and review of the literature. Vivo. 2014;28:311-4.

28. Mitchell A, Bendavid Y. Medullary colon cancer presenting with total necrosis of all regional lymph node metastasis: morphologic description of a presumed immune-mediated event. Diagn Pathol. 2014:9:204.

29. Jain S, Jain A, Onizuka N, et al. A rare case of medullary carcinoma of the colon presenting as intussusception in an adult with rectal bleeding. Hawaii J Public Health. 2014;73(348):52.

30. Kasapidis P, Grivas E, Papamichail V, et al. Medullary carcinoma of the colon: an adenoma with better prognosis. Ann Gastroenterol. 2015;28:289.

31. Wakasugi M, Kono H, Yasuhara Y, et al. A resected case of medullary carcinoma of the ascending colon followed by infarction of the greater omentum mimicking anastomotic leakage. Int J Surg Case Rep. 2017;41:456-60.

32. Martinotti M, Cirillo F, Ungari M, et al. Microsatellite instability in medullary carcinoma of the colon. Rare Tumors. 2017;9:6541.

33. Yago A, Momiyama M, Tateishi Y, et al. A case of medullary carcinoma of the ascending colon with intussusception. Nihon Rinsho Geka Gakkai Zasshi. 2018;79:1885-9.

34. Thirunavukarasu P, Sathaiah M, Singla S, et al. Medullary carcinoma of the large intestine: a population based analysis. Int $\mathrm{J}$ Oncol. 2010;37:901-7. 
35. Arai T, Takubo K. Clinicopathological and molecular characteristics of gastric and colorectal carcinomas in the elderly. Pathol Int. 2007;57:303-14.

36. Lynch HT, Smyrk T. Hereditary nonpolyposis colorectal cancer (Lynch syndrome). Cancer. 1996;78:1149-67.

37. Kinzler KW, Vogelstein B. Lessons from hereditary colorectal cancer. Cell. 1996;87:159-70.

38. Gonzalez RS, Cates JM, Revetta F, et al. Categorization and comparison with solid-type colonic carcinoma. Am J Clin Pathol. 2017;148:477-84.
Publisher's Note Springer Nature remains neutral with regard to jurisdictional claims in published maps and institutional affiliations. 